SCIENTIFIC REPORT

\title{
Elevations of AGE and vascular endothelial growth factor with decreased total antioxidant status in the vitreous fluid of diabetic patients with retinopathy
}

\author{
M Yokoi, S-i Yamagishi, M Takeuchi, K Ohgami, T Okamoto, W Saito, M Muramatsu, T Imaizumi, \\ S Ohno
}

Background/aims: Advanced glycation end product (AGE) induces vascular endothelial growth factor (VEGF) expression in cell culture and animal models, being considered to be involved in development of diabetic retinopathy; oxidative stress also has a part in diabetic retinopathy. However, the interrelations between AGE, VEGF, and oxidative stress remain to be elucidated. In this study, vitreous AGE, VEGF, and total antioxidant levels in were determined in diabetic patients with retinopathy, and the relations among them investigated.

Methods: ELISA (enzyme linked immunosorbent assay) was used to determine the vitreous levels of AGE and VEGF in 41 patients with diabetic retinopathy and 28 non-diabetic control subjects. Total antioxidant levels in vitreous of 20 diabetic patients and 18 controls were also analysed by ELISA.

Results: The vitreous levels of AGE and VEGF were significantly higher in diabetic patients than in control subjects ( $p<0.01$ for both). There was a significant correlation between the vitreous AGE and VEGF levels $(p<0.001)$. Total antioxidant status was decreased in vitreous in patients with diabetes compared with the controls $(p<0.01)$. Furthermore, both AGE and VEGF levels were inversely correlated with the total antioxidant status $(p<0.01$ and $\mathrm{p}<0.05$, respectively).

Conclusion: This study suggests that AGE and decreased total antioxidant status may contribute to the pathogenesis of diabetic retinopathy via induction of VEGF.

$\mathrm{R}$ educing sugars, including glucose, fructose, and trioses, can react non-enzymatically with the amino groups of proteins to form reversible Schiff bases, and then Amadori products. These early glycation products undergo further complex reactions such as rearrangement, dehydration, and condensation to become irreversibly cross linked, heterogeneous fluorescent derivatives termed advanced glycation end product (AGE). AGE can arise in vivo not only from glucose, but also from reducing sugars such as glyceraldehyde or methylglyoxal. ${ }^{2}$ The formation and accumulation of AGE is known to progress during normal ageing, and at an extremely accelerated rate in diabetes mellitus. ${ }^{12}$ Accumulating evidence shows a causal role for AGE in diabetic vascular complications. ${ }^{12}$ Indeed, AGE induces vascular endothelial growth factor (VEGF) expression in cell culture and animal models, thus being considered to be involved in the pathogenesis of diabetic retinopathy. ${ }^{3}{ }^{4}$ Furthermore, oxidative stress has also been reported to have a role in diabetic retinopathy. ${ }^{56}$ However, the interrelations between vitreous levels of AGE, VEGF, and oxidative stress in diabetic patients with retinopathy remain to be elucidated.

In this study, we used an enzyme linked immunosorbent assay (ELISA) to determine AGE, VEGF and total antioxidant levels in the vitreous fluid of patients with diabetic retinopathy, and investigated the relations among them.

\section{PATIENTS AND METHODS \\ Subjects}

The study involved patients with diabetes (20 men and 21 women) with a mean age of 53 (SD 12.6) years and a known mean duration of diabetes of 10.9 (8.5) years with a mean current $\mathrm{HbA}_{\mathrm{lc}}$ of $7.3 \%$ (1.3\%). The diagnosis of diabetes was made by the criteria of the American Diabetes Association reported in 1997. Based on the examinations by direct and indirect ophthalmoscopy, each subject's retinopathy was graded with respect to the severity of neovascularisation as diabetic macular oedema (DMO), proliferative diabetic retinopathy (PDR) with or without iris rubeosis. The severity scales of this study were appropriate because they were based on the previous reports of Freyberger et al or Bailey et al. ${ }^{8}$ DMO was defined as the presence of macular oedema without any of those including preretinal or disc neovascularisation or fibrovascular membrane, while PDR was defined as developing vitreous haemorrhage, preretinal or disc neovascularisation. Twenty eight non-diabetic patients (14 men and 14 women, mean age of 65.3 (SD 6.7) years) with idiopathic macular hole or epiretinal membrane served as control subjects. The study protocol was approved by the ethics committee of Hokkaido University School of Medicine, Sapporo, Japan. Informed consent was obtained from all subjects.

\section{Measurement of vitreous levels of AGE and VEGF}

During therapeutic vitrectomy, undiluted vitreous samples were obtained from 51 diabetic eyes, including six of DMO, 41 of PDR without iris rubeosis and four of PDR with iris rubeosis, and also from 28 control eyes. Vitreous levels of AGE and VEGF were measured with an ELISA as described previously. ${ }^{2}$ We measured glyceraldehyde derived AGE structure, which had been previously shown to induce VEGF expression both in cultured retinal pericytes and microvascular endothelial cells ${ }^{3}{ }^{9} ; 1 \mathrm{U}$ corresponds to $1 \mu \mathrm{g} /$ ml glyceraldehyde derived AGE-bovine serum albumin standard as described previously. ${ }^{2}$ When the vitreous levels

Abbreviations: $A G E$, advanced glycation end product; $D M O$, diabetic macular oedema; ELISA, enzyme linked immunosorbent assay; PDR proliferative diabetic retinopathy; VEGF, vascular endothelial growth factor 

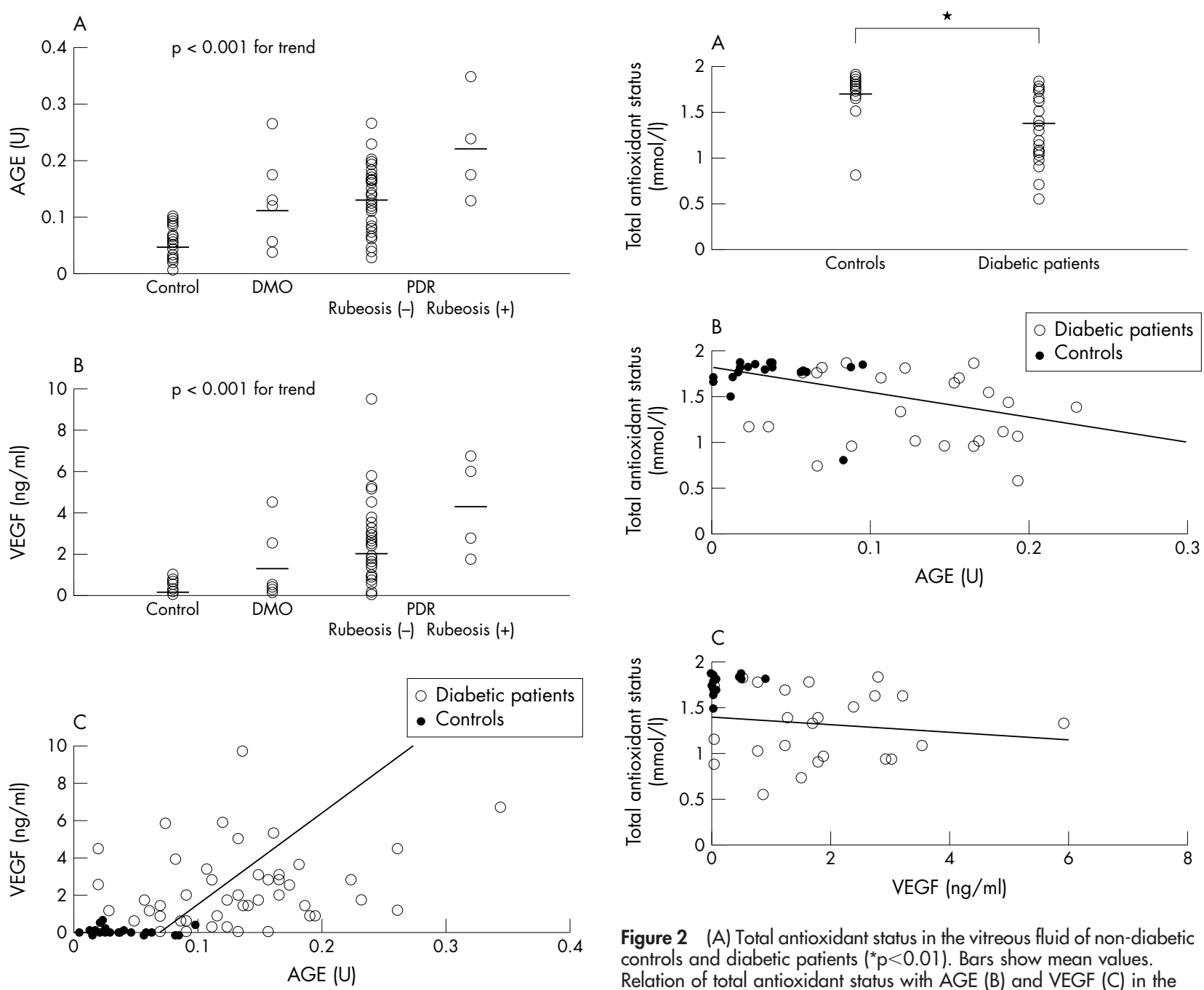

Figure 2 (A) Total antioxidant status in the vitreous fluid of non-diabetic controls and diabetic patients $\left({ }^{*} p<0.01\right)$. Bars show mean values. Relation of total antioxidant status with AGE (B) and VEGF (C) in the vitreous fluid of non-diabetic controls and patients with diabetic retinopathy. AGE and VEGF levels in the vitreous fluid were inversely

Figure 1 Correlation between the severity of neovascularisation in diabetic retinopathy and the vitreous levels of AGE (A) and VEGF (B). $A G E$ and VEGF levels in the vitreous fluid were significantly correlated with the severity of neovascularisation in diabetic retinopathy $(r=0.65$, $p<0.001$ and $r=0.68, p<0.001$, respectively). Bars show mean values. (C) Relation between the vitreous levels of AGE and VEGF in nondiabetic controls and patients with diabetic retinopathy $(r=0.59$, $\mathrm{p}<0.001$ ).

of the examined factors were under detection levels, we counted these values as zero.

\section{Measurement of total antioxidant levels in vitreous fluid}

Total antioxidant status in the vitreous fluid of 20 diabetic patients (25 eyes) and 18 controls (18 eyes) were also analysed with a commercially available ELISA kit (Calbiochem, La Jolla, CA, USA) according to the manufacturer's instructions.

\section{Statistical analysis}

Data were expressed as mean (SEM). Statistical significance was evaluated using Mann-Whitney $U$ test for paired comparison. Spearman's correlation coefficient by rank test was used for analysis of paired correlation, and for analysis between the severity of neovascularisation of diabetic retinopathy and vitreous levels of AGE and VEGF. A p value of less than 0.05 was considered to be significant. correlated with the total antioxidant status $(r=-0.46, p<0.01$ and $r=-0.37, p<0.05$, respectively).

\section{RESULTS}

\section{Vitreous levels of AGE and VEGF}

The vitreous levels of both AGE and VEGF were significantly higher in diabetic patients than in control subjects $(0.13$ (0.07) U $v 0.04(0.03) \mathrm{U}$ and $2.15(2.0) \mathrm{ng} / \mathrm{ml} v 0.12(0.23) \mathrm{ng} /$ $\mathrm{ml}$, respectively, $\mathrm{p}<0.01$ for both). As shown in figure $1 \mathrm{~A}$ and IB, vitreous levels of AGE and VEGF were associated with the severity of neovascularisation in diabetic retinopathy. Vitreous levels of AGE and VEGF were also higher in DMO patients than in non-diabetic controls $(0.13(0.08) \mathrm{U} v 0.04$ (0.03) $\mathrm{U}$ and 1.32 (1.73) ng/ml $v 0.12 \quad(0.23) \mathrm{ng} / \mathrm{ml}$, respectively, $\mathrm{p}<0.01$ for both). There was a significant correlation between the vitreous levels of AGE and VEGF $(r=0.59, \mathrm{p}<0.001$; fig $\mathrm{lC})$. There was no statistical correlation between the vitreous levels of AGE and $\mathrm{HbA}_{1 \mathrm{c}}$, patients' age, or duration of diabetes (data not shown). The serum levels of AGE were not correlated with those of vitreous AGE or VEGF (data not shown).

\section{Vitreous levels of total antioxidant}

Total antioxidant status in the vitreous was decreased in diabetic patients compared with control subjects ( $1.37(0.38)$ mmol $v 1.72(0.24) \mathrm{mmol}, \mathrm{p}<0.0 \mathrm{l}$; fig $2 \mathrm{~A})$. Furthermore, as shown in figure $2 \mathrm{~B}$ and $2 \mathrm{C}$, both AGE and VEGF levels were inversely correlated with the total antioxidant status in the 
vitreous fluid $(r=-0.46, \mathrm{p}<0.01$ and $r=-0.37, \mathrm{p}<0.05$, respectively). There was no significant correlation between the vitreous total antioxidant status and $\mathrm{HbA}_{\mathrm{lc}}$ levels (data not shown). Further, the serum levels of AGE were not correlated with the total antioxidant status in the vitreous fluid (data not shown). In a stepwise logistic regression analysis with PDR as the dependent variables including age, sex, diabetic duration, vitreous AGE levels, vitreous VEGF levels, and vitreous total antioxidant status as the independent variables, vitreous AGE levels had the strongest association with the development of PDR, but its association was not significant.

\section{DISCUSSION}

In this study, we demonstrated for the first time that vitreous levels of both AGE and VEGF were significantly higher in diabetic patients than in control subjects, and that their levels were elevated in association with the severity of neovascularisation in diabetic retinopathy. We also found here that vitreous levels of AGE and VEGF were correlated each other (fig lA-C). We, along with others, have previously shown that AGE induces VEGF expression in retinal cell culture and animals. $^{3}{ }^{4}$ Further, aminoguanidine, an inhibitor of AGE formation, prevents retinal VEGF overexpression in diabetic model animals. ${ }^{10}$ Our present study has extended these previous findings, showing that AGE may be one of the causal factors for VEGF induction in diabetic patients with retinopathy. In this study, serum levels of AGE were not associated with the severity of diabetic retinopathy (data not shown). Therefore, local formation and accumulation of AGE in diabetic retinas may play an important part in the progression of diabetic retinopathy in combination with VEGF. The findings that the vitreous fluid of patients with PDR contained enough levels of VEGF to promote angiogenesis $^{11}$ further support a pathological role for AGE elicited VEGF induction in the development of PDR.

VEGF is also known as a vascular permeability factor, and has been reported to be involved in the pathogenesis of DMO. ${ }^{12}$ In this study, we found that vitreous concentrations of AGE and VEGF in patients with DMO were higher than those in non-diabetic controls. Our results suggest that local accumulation of AGE could increase vascular permeability in diabetic macula via VEGF, thus being involved in the pathogenesis of DMO, in which vascular permeability is increased but proliferative change has not yet occurred. AGE induces blood-retinal barrier dysfunction by stimulating retinal VEGF expression in rats, supporting our speculation. ${ }^{1}$

In this study, we found for the first time that total antioxidant status was decreased in the vitreous of diabetic patients, and that vitreous levels of AGE and VEGF were inversely correlated with the total antioxidant status in patients with diabetic retinopathy (fig $2 \mathrm{~A}-\mathrm{C}$ ). The formation and action of AGE not only induces oxidative stress generation, but also inactivate a superoxide scavenging enzyme, Cu-Zn superoxide dismutase, both of which could lead to impairment of antioxidant defence systems. ${ }^{13}$ AGE may also contribute to the decreased total antioxidant status in diabetic retinopathy.

Oxidative stress also has an effect in diabetic retinopathy: it regulates vascular inflammation, gene expression of growth factors and cytokines, such as VEGF. ${ }^{56}$ Therefore, although it is not known whether the decrease in total antioxidant status is a case, consequence, or epiphenomenon, it is conceivable that the decreased antioxidant levels could further potentiate the deleterious effects of AGE on diabetic retinopathy through the overproduction of VEGF. Moreover, the formation, accumulation, and action of AGE are enhanced under oxidative stress conditions. ${ }^{1}$ These observations suggest that AGE and oxidative stress generation are correlated to form a positive feedback loop, thus having an important role in the development and progression of diabetic retinopathy.

The present study suggests that AGE and decreased total antioxidant status may contribute to the pathogenesis of DMO and PDR via induction of VEGF. Inhibition of AGE formation or oxidative stress generation could be a potential target for therapeutic intervention in sight threatening diabetic retinopathy.

\section{ACKNOWLEDGEMENTS}

This work was supported in part by grants of Venture Research and Development Centers from the Ministry of Education, Culture, Sports, Science and Technology, Japan (SY), and the Specific Research Fund of Hokuriku University, Japan (MT).

\section{Authors' affiliations}

M Yokoi, K Ohgami, T Okamoto, W Saito, M Muramatsu, S Ohno Department of Ophthalmology, Hokkaido University Graduate School of Medicine, Sapporo, Japan

S-i Yamagishi, T Imaizumi, Department of Internal Medicine III, Kurume University School of Medicine, Kurume, Japan

M Takeuchi, Department of Biochemistry, Faculty of Pharmaceutical Science, Hokuriku University, Kanazawa, Japan

Correspondence to: S Yamagishi, MD, PhD, Department of Internal Medicine III, Kurume University School of Medicine, Kurume 830-0011, Japan; shoichi@med.kurume-u.ac.jp

Accepted for publication 1 November 2004

\section{REFERENCES}

1 Yamagishi S, Takeuchi M, Inagaki Y, et al. Role of advanced glycation end products (AGEs) and their receptor (RAGE) in the pathogenesis of diabetic microangiopathy. Int J Clin Pharm Res 2003;23:127-32.

2 Takeuchi M, Makita Z, Bucala R, et al. Immunological evidence that noncarboxymethyllysine advanced glycation end-products produced from short chain sugars and dicarbonyl compounds in vivo. Mol Med 2000:6:114-25.

3 Yamagishi S, Amano S, Inagaki Y, et al. Advanced glycation end productsinduced apoptosis and overexpression of vascular endothelial growth factor in bovine retinal pericytes. Biochem Biophys Res Commun 2002;290:973-8.

4 Lu M, Kuroki M, Amano S, et al. Advanced glycation end products increase retinal vascular endothelial growth factor expression. J Clin Invest 1998;101:1219-24

5 Kowluru RA, Kennedy A. Therapeutic potential of anti-oxidants and diabetic retinopathy. Expert Opin Invest Drugs 2001;10:1665-76.

6 Hammes HP, Du X, Edelstein D, et al. Benfotiamine blocks three major pathways of hyperglycemic damage and prevents experimental diabetic retinopathy. Nat Med 2003;9:294-9.

7 Freyberger $\mathbf{H}$, Brocker $M$, Yakut $\mathrm{H}$, et al. Increased levels of platelet-derived growth factor in vitreous fluid of patients with proliferative diabetic retinopathy. Exp Clin Endocrinol Diabetes 2000;108:106-9.

8 Bailey CC, Sparrow JM, Grey RH, et al. The National Diabetic Retinopathy Laser Treatment Audit. III. Clinical outcomes. Eye 1999;13:151-9.

9 Okamoto T, Yamagishi S, Inagaki Y, et al. Angiogenesis induced by advanced glycation end products and its prevention by cerivastatin. FASEB J 2002;16:1928-30.

10 Frank RN, Amin R, Kennedy A, et al. An aldose reductase inhibitor and aminoguanidine prevent vascular endothelial growth factor expression in rats with long-term galactosemia. Arch Ophthalmol 1997; 115:1036-47.

11 Aiello LP, Avery RL, Arrigg PG, et al. Vascular endothelial growth factor in ocular fluid of patients with diabetic retinopathy and other retinal disorders. N Engl J Med 1994;331:1480-7.

12 Funatsu H, Yamashita H, Ikeda T, et al. Vitreous levels of interleukin-6 and vascular endothelial growth factor are related to diabetic macular edema. Am J Ophthalmol 2003;110:1690-6.

13 Arai K, Maguchi S, Fujii S, et al. Glycation and inactivation of human Cu-Znsuperoxide dismutase. Identification of the in vitro glycated sites. J Biol Chem 1987;262:16969-72. 